\title{
ESTUDO COMPARATIVO DE DATASETS GOVERNAMENTAIS DO BRASIL E DA COLÔMBIA, COM DADOS DE AGRICULTURA E DESENVOLVIMENTO RURAL
}

\author{
Jacquelin Teresa Camperos Reyes* \\ Universidade Estadual Paulista - UNESP, Brasil \\ Ricardo Cesar Gonçalves Sant'Ana** \\ Universidade Estadual Paulista - UNESP, Brasil \\ José Eduardo Santarém Segundo*** \\ Universidade de São Paulo - USP, Brasil
}

\begin{abstract}
Resumo: O trabalho analisa datasets de agricultura e desenvolvimento rural dos governos do Brasil e da Colômbia, questionando, a partir de princípios de dados abertos, se esses datasets realmente estão disponibilizados como dados abertos. Realizou-se análise de datasets observando aderência de alguns princípios de dados abertos criados em Sebastopol, cidade dos Estados Unidos. Os datasets do Brasil estão publicados em arquivos estruturados e não proprietários. A Colômbia tem tanto um número maior de datasets publicados quanto maior diversidade nos assuntos desses dados, no entanto, no que diz respeito ao Brasil, ainda deve apurar para que os dados estejam em formatos estruturados processáveis por máquina e não proprietários. Propõem-se novas pesquisas em torno de aprimorar mecanismos de avaliação de datasets governamentais, para verificar a abertura dos dados sob prismas como os de Sebastopol, até a criação de novos modelos, focalizando contribuições socioeconômicas que nasçam de pesquisas de profissionais da Ciência da Informação.
\end{abstract}

Palavras-chaves: Princípios de Sebastopol; dados abertos governamentais; datasets; dados Brasil; dados Colômbia.

Título: ESTUDIO COMPARATIVO DE DATASETS GUBERNAMENTALES DE BRASIL Y DE COLOMBIA, CON DATOS DE AGRICULTURA Y DESARROLLO RURAL.

Resumen: El artículo analiza datasets de agricultura y desarrollo rural de los gobiernos de Brasil y de Colombia, cuestionando, a partir de principios de datos abiertos, si esos datasets realmente están disponibilizados como datos abiertos. Se realizó análisis de datasets observando adherencia de algunos principios de datos abiertos creados en Sebastopol, ciudad de Estados Unidos. Los datasets de Brasil están publicados en archivos estructurados y no propietarios. Colombia tiene tanto un número mayor de datasets publicados, como mayor variedad en los asuntos de esos datos, sin embargo, con relación a Brasil, aún debe trabajar para que los datos estén en formatos estructurados procesables por máquina y no propietarios. Se proponen nuevas investigaciones en torno a perfeccionar modelos de evaluación de datasets gubernamentales, para verificar la apertura de los datos bajo prismas como los de Sebastopol, hasta la creación de nuevos modelos, enfocando contribuciones socio-económicas que nazcan de pesquisas de profesionales de la Ciencia de la Información.

Palabras clave: Principios de Sebastopol; datos abiertos gubernamentales; datasets; datos Brasil; datos Colombia.

Title: COMPARATIVE STUDY OF GOVERNMENT DATASETS OF BRAZIL AND COLOMBIA, WITH DATA OF AGRICULTURE AND RURAL DEVELOPMENT.

Abstract: This document analyzes agricultural and rural development datasets of the Brazilian and Colombian governments, questioning, from open data principles, if these datasets are indeed available as open data. It was performed an analysis of datasets observing the adherence of some of the open data principles created in the city of Sebastopol, United States. Brazilian datasets are published in structured and non-proprietary files. Colombia has both a greater number of published datasets and a greater variety in the subjects of such data, however, in relation to Brazil, it must still work in that the data is in machine-readable and non-proprietary structured formats. It is proposed to develop new researches aimed at the enhancement of evaluation mechanisms of government datasets, to verify the openness of the data under prisms like those of Sebastopol, even the creation of new models, focusing socioeconomic contributions that arise from researches of Information Science professionals.

Keywords: Principles of Sebastopol; open government data; datasets; Brazil data; Colombia data.

Copyright: (C) 2018 Servicio de Publicaciones de la Universidad de Murcia (Spain). Este es un artículo de acceso abierto distribuido bajo los términos de la licencia Creative Commons Reconocimiento 4.0 Internacional (CC BY $4.0)$.

\footnotetext{
*jtcamperos@marilia.unesp.br

***icardosantana@marilia.unesp.br

*** santarem@usp.br
}

Recibido: 25-08-2017; $2^{\mathrm{a}}$ versión: 26-02-2018; aceptado: 27-04-2018.

CAMPEROS REYES, J.T.; GONÇALVES SANT'ANA, R.C. y SANTARÉM SEGUNDO, J.E. Estudio comparativo de datasets gubernamentales de Brasil y de Colombia, con datos de agricultura y desarrollo rural. Anales de Documentación, 2018, vol. 21, $\mathrm{n}^{\circ}$ 2. Disponible en: http://dx.doi.org/10.6018/analesdoc.21.2.302381. 


\section{INTRODUÇÃ̃O}

A abertura dos dados governamentais é uma iniciativa que procura incrementar a participação cidadã na gestão da coisa pública, na qual seja possível acompanhar os processos governamentais dos Estados democráticos, favorecendo, assim, os ciclos de retroalimentação nas políticas públicas e seus resultados tangíveis nas sociedades que representam (Sant'Ana e Rodrigues, 2013). Ou seja, a participação cidadã nas democracias vai além da atuação dentro dos processos eleitorais no poder executivo, buscando o verdadeiro empoderamento desses grupos sociais.

Os dados abertos estão sendo observados como uma grande oportunidade dos governos no aprimoramento da sua relação com os cidadãos, tanto pela ampliação na disseminação de dados e informações a partir dos Estados, quanto na possibilidade de atender pedidos dessa natureza por parte de grupos sociais que buscam caminhos para suprir necessidades particulares.

No caso de economias em desenvolvimento, Verhulst e Young (2017) citam seis características como distintivas, as quais demarcam a importância no acesso e uso de dados publicados a partir de governos: possibilidade de escrutínio pela sociedade; igualdade na distribuição da informação; flexibilidade nos formatos de apresentação para a posterior utilização; amplo alcance de participação, tanto interna quanto na tomada de experiências fora do contexto regional; aumento da confiança por parte do grupo social representado pelo governo; e, amplificação do valor dos dados.

Os grupos sociais que acessam os dados mantêm características heterogéneas entre si, que trazem motivações diversas segundo o prisma utilizado para enxergar o conteúdo desses dados e informações (Gonzalez-Zapata e Heeks, 2015). Aponta Howard (2012) que nas iniciativas de dados abertos governamentais "Há muitas vocês diferentes", no que Ubaldi (2013) identifica a: os governos; cidadãos; sociedade civil; e, grupos econômicos, que vão desde a ampla economia, passando pela empresa privada, até chegar ao mercado de serviços públicos.

Nessa conjuntura, dados estão sendo disponibilizados na construção de governos abertos. Os dados abertos segundo a Open Knowledge Foundation (OKF) (2004) podem ser consumidos pela sociedade para utilizá-los, reutilizá-los ou redistribuí-los, contribuindo assim com o fortalecimento da atuação da sociedade mediante a visibilidade das ações governamentais (Santarém Segundo, 2013). Os dados abertos de governo são disponibilizados em sites oficiais mediante conjuntos de dados também chamados de datasets.

No entanto, por ser um processo ainda incipiente, precisa ser continuamente avaliado e acompanhado, neste caso, a partir da perspectiva de recomendações para a criação desses datasets. Neste texto, as recomendações que serão abordadas são os Oito Princípios de Sebastopol (Open Government Data, 2007).

O objetivo deste trabalho é analisar os datasets recuperados dentro dos domínios da Agricultura e Desenvolvimento Rural, nos sites de dados abertos dos governos do Brasil e da Colômbia, estudando-os por meio de princípios de dados abertos.

O Brasil tem tido destaque no desempenho agrícola mundial, tanto que a Organização das Nações Unidas para Alimentação e Agricultura (Food and Agriculture Organization of the United Nations - FAO) e a Organização para a Cooperação e Desenvolvimento Econômico (OCDE) (2015), ao realizar uma análise das perspectivas mundiais da agricultura, fizeram uma abordagem da atualidade global, dedicando maior interesse e estudo ao caso do Brasil. Destacam-se fatos como que o país é o responsável por mais da metade das exportações de açúcar a nível global, deslocou os Estados Unidos na liderança da produção de soja no mundo e fornece quantidades significativas de carnes, tabaco e milho. Configura-se assim o Brasil como uma referência para estudos sobre a temática.

A segunda referência é a Colômbia, com um potencial agrícola em 40,5\% do território nacional, ou seja, cerca de $462.000 \mathrm{~km}^{2}$ (Banco Mundial, 2014), o 100\% do território nacional dentro da franja da linha do Equador, e um sistema orográfico complexo que fornece variações da altitude, com suas consequentes pluralidades em temperaturas e umidades (Rangel-Ch e Aguilar-P, 1995), o que assinala à Colômbia como um país potencial na produção de alimentos.

Se bem ainda na atualidade há carência de sistemas e métricas que permitam quantificar certeiramente o impacto e a animação nas economias, junto às relações causais, da disponibilização de dados a partir de governos (Abella; Ortizde-Urbina-Criado e De-Pablos-Herdero, 2014 e Huijboom y Van den Broek, 2011), no setor da agricultura e a nutrição, preveem-se esses quatro impactos: melhoria na governança; oportunidade de inovação e criação; soluções a problemas públicos; e, empoderamento da cidadania (Verhulst e Young, 2017). 
Apresenta-se neste trabalho uma reflexão sobre a aplicação dos oito princípios de Sebastopol em datasets publicados pelos governos do Brasil e da Colômbia, dentro do domínio da Agricultura e Desenvolvimento Rural. Percebeu-se no desenvolvimento da pesquisa que essas recomendações feitas na reunião de Sebastopol, em 2007, que deveriam ser acatadas pelos responsáveis da elaboração de datasets governamentais, ainda estão em processo de implementação nos governos do Brasil e da Colômbia.

Considera-se relevante a utilização de recomendações como as de Sebastopol por parte das entidades e instituições responsáveis pela publicação dos datasets, quando foi evidenciado na pesquisa que os princípios analisados estão sendo aplicados parcialmente, elucidando que, para cada um deles, existem diferenças nos processos particulares de cada país. A exemplo disso observou-se nos resultados que no Brasil 100\% dos arquivos usados para disponibilizar datasets encontram-se em formatos estruturados, enquanto, na Colômbia, somente apresentaram-se em $78 \%$ dos datasets objeto desta pesquisa.

\section{CIRCUNSTÂNCIAS DE DADOS NOS DOMÍNIOS DA AGRICULTURA E DESENVOLVIMENTO RURAL}

Um fato tecnológico agiu com destaque no uso de dados abertos, o Global Positioning System (GPS), sistema criado com o fim de conhecer a localização exata de uma entidade física ou pessoa mediante navegação espacial.

Esta tecnologia, embora inicialmente restrita às forças militares nos Estados Unidos, passou a ser aberta, permitindo usos fundamentais dos dados (medições) pela economia, tendo a agricultura como um dos setores que apropriou-a ao seu favor. Uma das implementações tem se dado no surgimento da agricultura de precisão, a qual utiliza como instrumentos tecnológicos aparelhos com GPS (Young e Verhulst, 2016).

Segundo Lamparelli (201?), a agricultura de precisão é um “[...] conjunto de técnicas que permitem o gerenciamento localizado dos cultivos" utilizando ferramentas tecnológicas, na busca de rentabilidade e redução de custos agrícolas. Uma pesquisa desenvolvida por Nardo (2015) no estado de São Paulo no Brasil, deu como um dos resultados, que o uso da agricultura de precisão para o planejamento de cenários de plantio de cana-de-açúcar, obtém uma melhoria no tempo de manobra na ordem de $25,6 \%$, com a consequente diminuição no consumo de combustíveis, e uma melhora de $34,95 \%$ nas linhas de plantio, aprimorando assim, a eficiência operacional do campo.

Outro uso de dados abertos, deu-se com a liberação de dados meteorológicos por parte da Administração Nacional Oceânica e Atmosférica dos Estados Unidos (em inglês - National Oceanic and Atmospheric Administration NOAA), a qual conquistou uma mudança paradigmática na gestão das lavouras, por meio de dados relacionados à previsão do tempo. A NOAA ofereceu dados meteorológicos que pudessem ser utilizados por setores como a indústria agrícola. Na prática, essa implementação obteve um benefício econômico que superou os U\$460 milhões em razão ao uso dos dados na tomada de decisões durante os anos que ocorreram os fenômenos climatológicos El Niño e La Niña (Young e Verhulst, 2016).

Como fato político, a Organização das Nações Unidas (ONU) reunida em 2015 determinou a criação da Agenda 2030 para o Desenvolvimento Sustentável ${ }^{1}$, composta por 17 metas que constituem o embasamento do plano de ação que busca a transformação do panorama mundial (ONU, 2017).

A segunda meta deste plano de ação é End hunger, achieve food security and improved nutrition and promote sustainable agriculture. Diante da crise que sofrem as povoações famintas e com a segurança alimentar em risco, inicialmente focadas no continente da África, porém espalhadas nos outros quatro continentes, a ONU (2017) espera reduzir a fome extrema, aspecto que considera um grande obstáculo no desenvolvimento humano e por tanto, no desenvolvimento sustentável. Na atualidade existem 748 milhões ${ }^{2}$ de pessoas que estão nessa situação.

Ainda, desde o ano 2012, a cúpula dos oito países mais desenvolvidos do mundo, chamada o G8 $8^{3}$, determinou o compartilhamento dos dados relacionados com a agricultura e a nutrição que fossem gerados nesses países, a saber: Estados Unidos, Grã-Bretanha, Canadá, França, Alemanha, Itália, Japão e Rússia. Isto com a intenção de que os países em desenvolvimento conseguissem utilizar esses dados, na busca da melhora dos processos internos, proporcionando auxílio tanto na geração de alimentos quanto na redução da pobreza. 
A importância dita com relação aos dados dentro do domínio da agricultura e desenvolvimento rural, fundada em fatos sociais, políticos e tecnológicos, instiga à exploração dos atuais datasets de governos e das características com as quais eles estão sendo disponibilizados.

\subsection{Publicação de dados nos países da pesquisa}

No contexto mundial da publicação de dados oficiais, a Colômbia tem obtido destaque na implementação de estratégias que visam impulsionar a publicação de dados a partir do governo nacional (OCDE, 2017).

A OCDE desenvolveu The OCED OURdata Index, um marco de trabalho que reúne indicadores na temática de dados no governo aberto. O marco mede esforços desenvolvidos para estimular a abertura e promoção no uso de dados, propendendo pela reutilização entre cidadãos e empresas, resumindo pontos fortes e fracos, e identificando áreas de ação nas políticas nacionais dos países membros da OCDE (2017).

Na última medição do OURdata Index, com dados pesquisados entre novembro e dezembro de 2016, mensuraramse os esforços desenvolvidos pelos governos para a promoção, uso, reuso e disponibilização de ferramentas para a publicação de dados. Nessa edição a Colômbia aparece em quarto lugar no ranking global, que tem em seus primeiros colocados, a Coreia do Sul, a França e o Japão. O índice obtido pela Colômbia foi de 0,804 (OCDE, 2017).

O Brasil é um dos países pioneiros da América Latina e do mundo na promoção e execução de políticas de Estado para o uso de dados, com repercussão e convocatória para outros países. Mostra disso foi a participação na primeira reunião da Open Government Partnership ${ }^{5}$ (OGP), acontecida no ano 2011 (OGP, 2017).

Nesse encontro o Brasil, além de fazer parte do grupo de oito países pioneiros ${ }^{6}$, partilhou a presidência com os Estados Unidos, e voltou-se, junto ao México, países latino-americanos que se oficializaram como pioneiros na estratégia de abertura de ativos informacionais de governos ${ }^{7}$. O índice obtido pelo Brasil no último OURdata Index realizado no ano 2017 foi de 0,54 (OCDE, 2017). Cabe salientar o desempenho da Espanha no índex dito, sendo de 0,76, colocando o país ibérico numa posição de destaque, apenas superado por dois Estados europeus, a França e a Grã-Bretanha (OCDE, 2017).

Tanto no Brasil quanto na Colômbia as iniciativas para a abertura dos dados governamentais têm trazido ao cenário novas organizações, modificando assim parte das estruturas dos governos. Essas organizações têm sob tutela processos e atividades relacionadas com a disponibilização de dados e informações.

No Brasil, o Ministério do Planejamento, Orçamento e Gestão (MP), por meio da Secretaria de Logística e Tecnologia da Informação, instituiu em 2012 a Infraestrutura Nacional de Dados Abertos (INDA), como uma política do governo que garanta o acesso aos dados e às informações geradas e custodiadas pelo Poder Executivo Federal.

A INDA é uma das realizações concretas do governo federal logo após da primeira reunião mundial para o Governo Aberto de 2011, na qual, o Brasil foi um dos países partícipes. Esta política transcorre dentro da instrumentalização da Lei de Acesso à Informação 12.527 de 2011 (Brasil, 2011).

A Instrução Normativa $N^{\circ} 4$ de 2012 do MP, indicou que a INDA seria gerenciada por um comitê gestor integrado pelos representantes do governo, pela sociedade civil e pelo setor acadêmico. Os representantes do setor do governo serão da Secretaria de Logística e Tecnologia da Informação, do Ministério do Planejamento, Orçamento e Gestão; da Casa Civil da Presidência da República; da Controladoria Geral da União; do Ministério da Ciência, Tecnologia e Inovação; do Ministério do Desenvolvimento Social; do Ministério da Educação; do Ministério da Saúde; a Secretaria Geral da Presidência da República; e do Instituto Brasileiro de Geografia e Estatística (IBGE) (Brasil, 2012).

A INDA é a responsável por realizar a gestão da política pública brasileira dos dados abertos, indicando as diretrizes para sua implementação, disponibilizando as tecnologias necessárias, os procedimentos e os padrões nos processos de publicação. Ela tem como principal projeto o site $<$ dados.gov.br $>$, que funciona como ponto central para a publicação, a busca e o acesso a dados públicos (Santarém Segundo, 2015 e Brasil, 2012).

No caso da Colômbia, a infraestrutura dos dados abertos do governo é administrada pelo Ministerio de Tecnologías de la Información y las Comunicaciones, dentro da estratégia chamada Gobierno en Línea. Assim, foi criado o site < datos.gov.co> desde 2011, mantendo ali informações necessárias para os usuários que pretendem tanto publicar datasets quanto os que desejam pesquisar os disponibilizados. Aliás, é possível consultar outro tipo de informações relacionadas com essa estratégia tais como estatísticas do uso dos datasets e convocatórias diversas sobre o uso de 
dados abertos. Este site age ainda como canal de comunicação com seus diferentes perfis de usuários, permitindo o envio de mensagens com solicitudes de novos tipos de conjunto de dados, bem como dados que ainda estejam no processo de disponibilização.

Esta plataforma governamental criada durante a implementação da lei colombiana 1712 de 2014, chamada Lei de Acesso à Informação, está fundamentada na interação de órgãos do governo que fornecem apoio em aspectos estratégicos, jurídicos, implementares, de sensibilização, capacitação e ensino, e convergem assim, seguindo o Plano de Ação da estratégia Gobierno en Línea (Colômbia, 2015).

Em 2015 o Ministerio de Tecnologías de la Información y las Comunicaciones da Colômbia, por meio de uma cooperação técnica com o Banco Mundial (BM), realizou um diagnóstico dos avanços na abertura dos dados, com o fim de fornecer uma ferramenta de avaliação e de reflexão em torno a este processo. O documento resultante desse diagnóstico, realizado mediante a metodologia Open Data Readiness Assessment (ODRA) do BM, entre seus resultados apresenta uma compilação da interação das instituições indicada no parágrafo precedente, representado na Figura 1 (Colômbia, 2015).

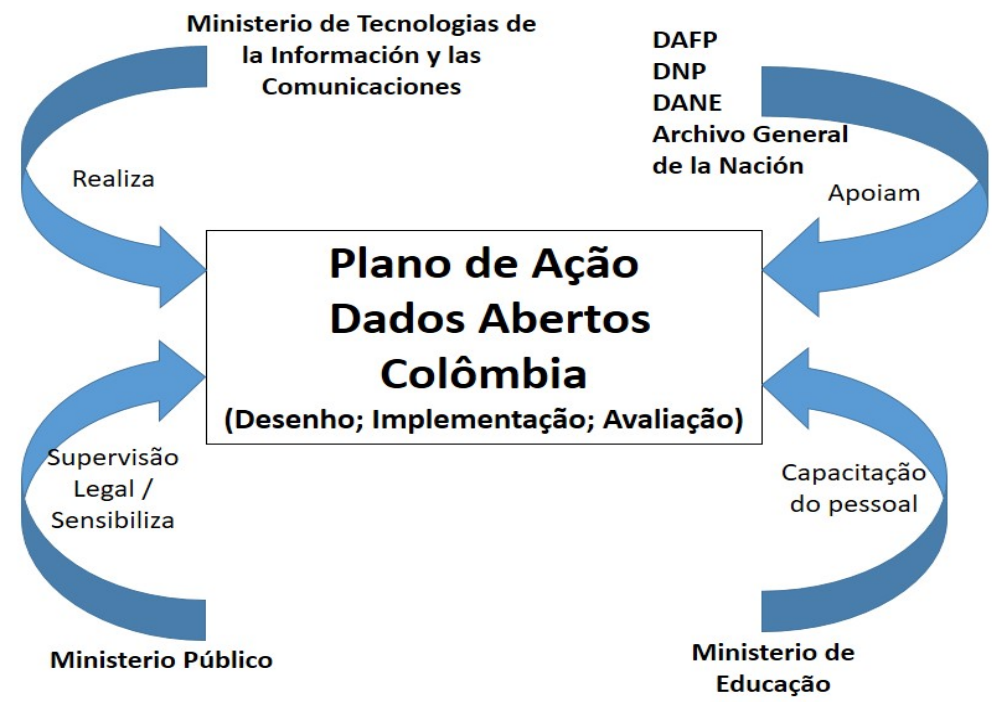

Figura 1. Interação de órgãos do governo da Colômbia no Plano de Ação de Dados Abertos. Fonte: Adaptado de Colômbia (2015).

Tentando observar pontos de similaridade entre os dois países por meio da metodologia ODRA, pesquisou-se dito estudo no Brasil, tanto no portal de dados brasileiros, quanto no site do grupo de dados abertos do BM, http://opendatatoolkit.worldbank.org/en/odra.html, encontrando que para o Brasil ainda não tem sido publicado o resultado da aplicação dessa metodologia. Em um acordo de cooperação técnica entre o BM e a OKF capítulo Brasil, implementou-se a ODRA unicamente no âmbito do governo do Distrito Federal ${ }^{8}$ (OPEN KNOWLEDGE BRASIL, 2014).

Ainda no contexto latino-americano, e com antecedência à Colômbia, México, Peru e República Dominicana também acolheram a metodologia ODRA com o intuito de colaborar em cada governo com a abertura de dados. Nesses casos a ODRA realizou-se como estudo de preparação, pensando em identificar ações previas a serem consideradas para lançar a iniciativa Open Data em cada um desses Estados (México, 2013; Peru, 2013 e República Dominicana, 2014).

No âmbito espanhol, com o propósito de mensurar o grau de reutilização ${ }^{9}$ de dados e informações fornecidas em datasets por órgãos oficiais, criou-se a Métrica para evaluar la reutilización de datos abiertos (MELODA). Este mecanismo de avaliação observa seis dimensões nos datasets de governo: padrões técnicos, acesso, legal, modelos de dados, informação geolocalizada, e, informação em tempo real (Abella; Ortiz-de-Urbina-Criado e De-PablosHeredero, 2014 e Abella, 2016); focando uma forma quantitativa de conhecer as condições de acesso e reutilização da informação em instâncias do governo espanhol. 


\section{PROPOSTA DE AVALIAÇÃO À LUZ DOS OITO PRINCÍPIOS DE DADOS ABERTOS DE SEBASTOPOL}

Dados abertos referem-se aos dados surgidos dentro de um movimento que promove a liberação de dados a partir das instituições públicas, com a particularidade de estar representados de tal forma que seja válida sua reutilização (Peset; Ferrer-Sapena e Subirats-Coll, 2011), num entorno onde a transparência aja como meio que coadjuve na geração de dados verdadeiramente úteis, até para serem usados como fonte de inovação (Attard et al., 2015).

No ano 2007, em Sebastopol nos Estados Unidos, realizou-se um encontro de trinta ativistas defensores do movimento de governo aberto. Nesse encontro, o grupo de trabalho refletiu sobre as características que deveriam ter os dados publicados pelos governos que estavam aderindo-se ao modelo de governo aberto, ou seja, publicando dados abertos, para assim, cumprir com o objetivo de servir à sociedade na disponibilização dos dados para o uso e o reuso (Open Government Data, 2007).

Em consonância com a Open Knowledge Foundation (2004) com relação à utilização, reutilização e distribuição de dados de governos, no leque das opções da internet, como meio de comunicação, o grupo de trabalho de Sebastopol, destacou a grande oportunidade dos governos no aprimoramento da sua relação com os cidadãos, tanto pela ampliação na disseminação de dados e informações a partir dos Estados, quanto na possibilidade de atender pedidos dessa natureza por parte de grupos sociais que buscam caminhos para suprir necessidades particulares.

Como resultado do encontro, foram propostos os Oito Princípios de Dados de Governo Aberto, conhecidos como Os Oito Princípios de Sebastopol (Open Government Data, 2007). Eles surgem com o intuito de estabelecer um marco geral das características que devem ter os dados abertos publicados pelos governos, isto é, os datasets com dados governamentais. Para que os dados de um governo sejam publicados como dados abertos, devem respeitar os seguintes princípios:

\section{Completo}

Todos os dados públicos devem ser disponibilizados; dados públicos para serem publicados que não estejam sujeitos a limitações de privacidade, segurança ou privilégios especiais.

O princípio refere-se a que os datasets publicados contenham TODOS os dados que o órgão oficial tenha gerado sobre o tema ou assunto. A justificativa para que dados e informações não estejam publicados, unicamente deve corresponder com políticas de segurança e privacidade de cada Estado.

2. Primário

Os dados estão tal como foram recolhidos na fonte, com o mais alto nível possível de granularidade, e não somente em forma agregada nem modificada. Os órgãos do governo devem evitar a transformação dos dados, isto é, o tratamento com processos que modifiquem sua essência e que façam que os dados sejam apresentados de forma generalizada, condensada ou resumida, perdendo assim a sua granularidade.

3. Oportuno

Os dados são disponibilizados tão rapidamente quanto necessário para preservar o valor temporal dos dados. Recomenda que os dados sejam disponibilizados o mais próximo possível a data da coleta.

\section{Acessível}

Os dados estão disponíveis para a mais ampla gama de usuários e propósitos. Disponibilizam-se sem discriminação tanto a partir da ótica tecnológica, desconsiderando restrições inerentes às tecnologias de acesso, quanto com relação aos propósitos atuais e futuros dos usuários dos dados.

5. Processáveis por máquina

Os dados são estruturados com o fim de permitir o processamento automatizado. Isto inclui processos de codificação para os dados outorgando estruturas que permitem o seu processamento. $\mathrm{O}$ fato de publicar dados sem estrutura, mesmo que completos, oportunos e accessíveis, torna seu processamento automatizado complexo. Esse princípio tem incidência com as ponderações sobre os padrões e esquemas de metadados dos datasets.

6. Não discriminatório

Os dados estão disponíveis para qualquer pessoa sem necessidade de registro, ou seja, usos anônimos devem ser permitidos até por proxies $w e b^{10}$, em qualquer tempo e sem especificar justificativa para o acesso e o uso.

7. Não proprietário

Os dados estão disponíveis em um formato no qual nenhuma entidade tem controle exclusivo. Com arquivos em formatos proprietários, ao tentar acessar os dados, requerem-se aplicativos específicos que não são de domínio público. Esses aplicativos adicionam restrições à origem do acesso aos dados, à temporalidade do uso e às formas de uso desses dados.

8. Livre de Licença 
Os dados não devem estar sujeitos a qualquer direito autoral, patente, marca registrada, nem segredo comercial. Uma privacidade razoável é admissível, considerando razões de segurança do governo. Recomenda-se especificar em todos os casos o tipo de licença e restrições legais que se aplicam aos dados disponibilizados.

Estes oito princípios descritos agem como identificadores sobre se os dados que estão sendo publicados pelos governos, podem considerar-se como abertos ou não, sendo assim necessário um olhar a partir do viés de cada princípio nos datasets publicados.

\subsection{Método da estimação}

Considerando o objetivo do trabalho realizou-se pesquisa descritiva, examinando os sites que publicam dados governamentais, dados.gov.br do Brasil, e <datos.gov.co> do governo da Colômbia, focando nos datasets de Agricultura e Desenvolvimento Rural. A pesquisa é classificada como um Estudo de Caso pela abordagem de datasets disponíveis em razão do detalhamento dos seus dados diante dos princípios de Sebastopol.

No site <dados.gov.br> do Brasil, a pesquisa foi feita pelos termos Agricultura, e, Desenvolvimento Rural, na caixa de busca da tela principal. No caso da Colômbia, existe uma categoria intitulada Agricultura e Desenvolvimento Rural.

A consulta nos dois sites foi realizada no período compreendido entre 1 e 19 de agosto de 2016, analisando os datasets recuperados em cada site atendo os sequentes critérios de avaliação.

No caso do princípio 1: Completo, um cenário fatível de avaliação, faz pensar na existência prévia de um inventario total dos dados gerados pelos órgãos nos governos, dados que são susceptíveis de disponibilização, isto é, que não estejam sujeitos a restrições de privacidade e segurança nacional.

Para abordar o princípio 2: Primário, a granularidade presente em um dataset, deve-se considerar que ela está relacionada com a possibilidade de obter subconjuntos abstraídos a partir de critérios de dissimilaridade dentro deles (Santos e Santana, 2015), o qual permitiria o tratamento diversificado dos dados recuperados, devido ao nível de detalhamento com que os dados se publicam. A granularidade pode ser categorizada segundo Alves, Simionato e Santos (2012) em dois níveis: granularidade fina e granularidade grosa.

Para o princípio 3: Oportunidade, temos que na publicação de datasets, existe uma relação de dependência dos tempos tanto da instituição que disponibiliza dados, como da data de publicação no portal de dados abertos. Trata-se de ações que ocorrem de modo autônomo por parte de cada órgão ou instituto de governo.

Com o anteriormente exposto, percebe-se que no caso dos princípios de Sebastopol 1, 2 e 3, têm-se ausência de critérios específicos que permitam mensurar o cumprimento de cada princípio. Já para analisar de modo específico os princípios 4, 5, 6, 7 e 8, é possível realizar uma estimação dentro dos datasets objetos desta pesquisa considerando os tópicos abordados no Quadro I.

\begin{tabular}{|l|l|}
\hline Princípio & Características a observar nos datasets \\
\hline 4 Acessível & $\begin{array}{l}\text { Identificação dos tipos de usuários que podem acessar, } \\
\text { a existência de restrições sobre o tipo de sistema } \\
\text { operativo (com eles toda a gama de hardware) que } \\
\text { pode acessar, os navegadores web permitidos, e } \\
\text { especificação do tipo de uso futuro dos dados } \\
\text { disponibilizados }\end{array}$ \\
\hline $\begin{array}{l}\text { 5 Processáveis por } \\
\text { máquina }\end{array}$ & $\begin{array}{l}\text { Quais os formatos dos arquivos presentes nos datasets } \\
\text { recuperados }\end{array}$ \\
\hline 6 Não discriminatório & $\begin{array}{l}\text { Observar a exigência de registro prévio para } \\
\text { possibilitar aos usuários consultarem os dados } \\
\text { publicados }\end{array}$ \\
\hline Identificar os formatos dos arquivos presentes nos \\
datasets, indicando se são arquivos proprietários ou \\
não proprietário
\end{tabular}

Quadro I. Critérios de avaliação para os princípios 4, 5, 6, 7 e 8. Fonte: Adaptado de Open Government Data (2007). 
Para a análise proposta os datasets encontrados foram sistematizados a fim de classificar e descrevê-los considerando: assunto do dataset (com fins informativos); perfis de usuários com permissão para a consulta; formatos de arquivos presentes nos datasets; requisição ou não de registro de usuário prévio para a consulta; formatos de arquivos proprietários ou não; e, a presença e o tipo de licença nesses datasets.

\section{RESULTADOS}

No site do Brasil foram encontrados trinta e dois (32) datasets; no caso da Colômbia, o total de datasets recuperados foi setenta (70). Apresenta-se a análise:

\section{Princípio Completo}

Ambos os governos, do Brasil e da Colômbia, estão incluindo cada vez mais novos datasets nos seus sites, melhorando assim a oferta de dados para a cidadania em geral. No caso da Colômbia, está em andamento o processo de publicação dos "Registros de Ativos de Informação" (Colômbia, 2014, p. 7, tradução nossa) por parte das entidades sujeitas à disponibilização de dados abertos. Embora a Colômbia esteja publicando dados abertos desde 2011, e como parte de um processo de melhora continua, ainda está em andamento a identificação da oferta de datasets gerada nesse governo (Colômbia, 2014 e Colômbia, 2016).

Percebeu-se que no Brasil e na Colômbia, não existe a possibilidade de determinar se os datasets recuperados na data da pesquisa foram publicados como dados Completos, ou seja, se correspondem ao cem por cento dos dados existentes nos órgãos de governo que publicaram esses dados nos países.

\section{Princípio Primário}

Quando a Open Government Data indica o fundamento deste princípio, refere tanto a qualidade granular dos dados, quanto os processos de transformação que alterem a essência dos dados publicados, com isso, para avaliar o princípio Primário mediante mensuração dessas propriedades nos datasets, faz-se necessário ter conhecimento do domínio de cada instituição que esteja publicando dados, para assim chegar a compreender por um lado, se os datasets têm o nível de granularidade fina que permita gerar possíveis subconjuntos de dados dentro de uma dimensão determinada, e do outro, o estado original dos dados na fonte para dessa maneira inferir as possíveis alterações que sofreram esses recursos.

\section{Princípio Oportuno}

No marco legal do Brasil, indica-se que os procedimentos de disponibilização de dados e informações deverão ser observados pelos próprios órgãos públicos, além das entidades privadas que administrem recursos públicos (Brasil, 2011). Determinar se os datasets têm sido publicados dentro dos prazos particulares de cada instituição tanto brasileira quanto colombiana, implicaria sair do contexto da publicação de datasets nos sites de dados e adentrar-se aos procedimentos de cada instituição, examinando seus processos internos de publicação de dados abertos.

\section{Princípio Acessível}

O cem por cento dos datasets encontrados tanto no site do Brasil quanto o da Colômbia não precisam de nenhuma classe de identificação relacionada com perfil de usuário, que possibilite ou não o acesso, assim como também não é solicitado identificar o uso futuro dos dados. Não foi observada nenhuma restrição enquanto aos sistemas operativos e navegadores que podem acessar esses datasets.

\section{Princípio Processáveis por Máquina}

Os datasets recuperados nos dois países se encontram disponíveis nos formatos indicados no Quadro II. 


\begin{tabular}{|c|c|c|c|}
\hline \multicolumn{4}{|c|}{ CLASSIFICAÇÃO DOS TIPOS DE ARQUIVOS } \\
\hline Categoria & Estruturados & Empacotados & Outras formas divulgação \\
\hline \multirow{5}{*}{ 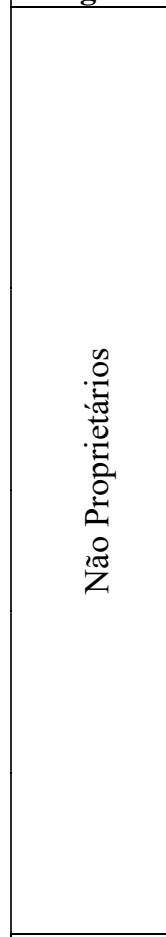 } & $\begin{array}{l}\text { XML } \\
\text { (eXtensible Markup Language) } \\
\text { Linguajem de marcado que fornece } \\
\text { formato simples baseado em texto } \\
\text { para estruturar informação. Derivou } \\
\text { do formato SGML, adequando-se } \\
\text { para o uso na web (W3C, 2017). }\end{array}$ & $\begin{array}{l}\text { KMZ } \\
\text { Arquivos que } \\
\text { permitem empacotar } \\
\text { vários arquivos juntos. }\end{array}$ & $\begin{array}{l}\text { HTML } \\
\text { (HyperText Markup Language) } \\
\text { É uma linguagem de marcação, } \\
\text { usado para criar páginas web e } \\
\text { elementos que conformam websites } \\
\text { (W3C, 2017) }\end{array}$ \\
\hline & $\begin{array}{l}\text { JSON } \\
\text { (JavaScript Object Notation) } \\
\text { Formato leve de troca de dados da } \\
\text { linguagem JavaScript. }\end{array}$ & $\begin{array}{ll}\text { ZIP } & \\
\text { Formato } & \text { de } \\
\text { compressão } & \text { de } \\
\text { arquivos } & \end{array}$ & $\begin{array}{l}\text { RSS } \\
\text { (Really Simple Syndication) } \\
\text { Arquivo em formato XML usado } \\
\text { para compartilhar informação na } \\
\text { web }\end{array}$ \\
\hline & $\begin{array}{l}\text { GEOJSON } \\
\text { Formato para codificação de dados } \\
\text { geográficos. }\end{array}$ & & \\
\hline & $\begin{array}{l}\text { RDF } \\
\text { (Resource Description Framework) } \\
\text { Marco de trabalho para à descrição } \\
\text { de informação na web (W3C, 2017) }\end{array}$ & & \\
\hline & $\begin{array}{l}\text { CSV } \\
\text { (Comma separated values) } \\
\text { Arquivo de valores separados por } \\
\text { virgulas }\end{array}$ & & \\
\hline 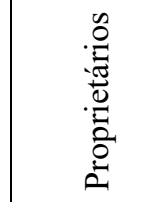 & $\begin{array}{l}\text { XLS } \\
\text { Arquivo tipo planilha. Arquivo } \\
\text { proprietário da empresa Microsoft. }\end{array}$ & & \\
\hline
\end{tabular}

Quadro II. Formatos de arquivos disponíveis nos datasets recuperados. Fonte: Elaborado pelos autores.

Tendo em conta que o princípio Processável por Máquina faz referência a arquivos estruturados que permitam o processamento automático dos dados, dos formatos descritos no quadro II, quem têm essa característica são: o JSON; o XML; o GEOJSON; o XLS; o CSV; e o RDF.

A Figura 2 apresenta a distribuição dos formatos de arquivos nos datasets recuperados no Brasil.

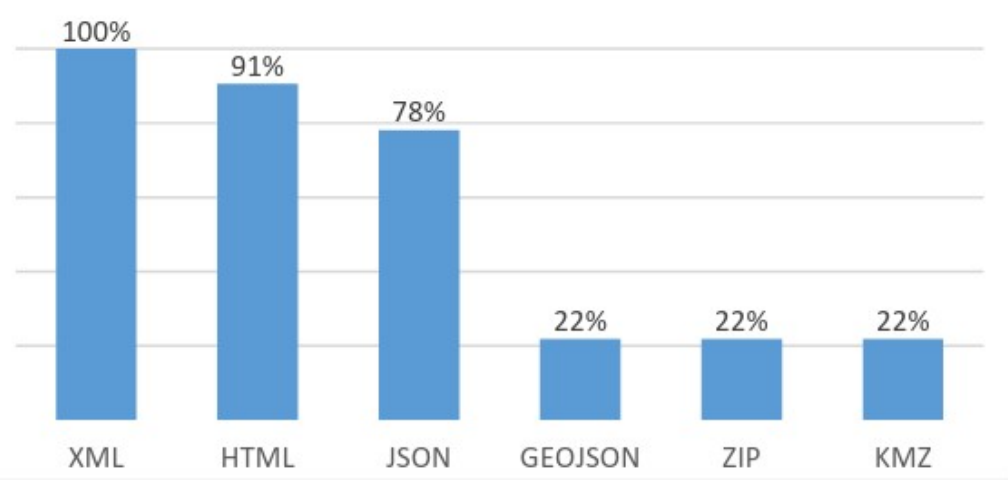

Figura 2. Distribuição dos datasets segundo o formato de arquivo - Brasil. Fonte: Elaborado pelos autores.

A totalidade dos datasets disponibilizados no âmbito da pesquisa, estão em pelo menos um tipo de formato estruturado, o XML, mostrando a boa aderência dessa linguagem de marcação no governo brasileiro. Ainda, além do formato XML presente em todos os datasets, a maioria deles estão disponibilizados em mais um tipo de formato estruturado: JSON ou GEOJSON. 
Em seguida, o formato JSON está presente nos 78\% dos datasets analisados. O Manual dos Dados Abertos para Brasil, com autoria do World Wide Web Consortium (W3C) (2009), indica a facilidade no uso e na interpretação pelas linguagens de programação dos arquivos JSON.

Ressalta-se que existem datasets publicados em HTML, disponibilizados como uma segunda alternativa aos formatos XML e/ou JSON, entendendo que se publica em HTML com o fim de dar uma visualização gráfica por conta da natureza dos dados, que nesses datasets corresponde-se com dados geográficos.

Gertrudis-Casado, Gértrudix-Barrio e Álvarez-García (2016) referem que apesar de que com fins didáticos os datasets podem apresentar-se mediante visualizações interativas, os dados, devem estar dispostos em formatos reutilizáveis, isto é, em formatos estruturados, fato que está evidenciado no grupo de datasets recuperados no site brasileiro.

Ainda, na amostra do Brasil, datasets publicados nos formatos ZIP e KMZ, formatos de arquivos agregados, são publicados como alternativas a arquivos estruturados tais como XML, GEOJSON ou JSON.

O gráfico de barras da Figura 3, elaborado segundo os tipos de formatos de arquivos encontrados nos datasets da Colômbia, representa a distribuição nos 70 datasets recuperados.

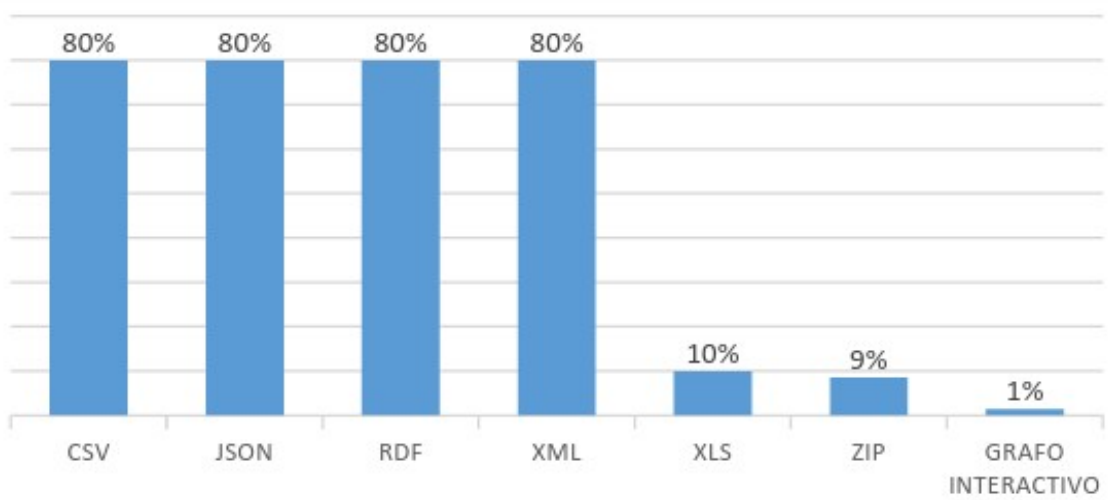

Figura 3. Distribuição dos datasets segundo formato de arquivo - Colômbia. Fonte: Elaborado pelos autores.

Avaliando o quinto princípio no grupo de datasets recuperados no site da Colômbia, observou-se que do universo analisado, $80 \%$ desses datasets encontram-se em formatos de arquivos estruturados, o que deixa uma fração importante de dados sem a possibilidade de ter processamento automatizado por parte dos usuários do site de dados colombiano. Essa porcentagem de arquivos é composta por 56 datasets que estão publicados nos formatos CSV, JSON, RDF e XML.

Os datasets expostos em XLS, nos quais é possível fazer processamento automático, os usuários ficam dependendo das estruturas dos arquivos, que podem em algum momento ser modificadas pelos responsáveis desses dados. Sete datasets da amostra da Colômbia foram recuperados com publicação exclusiva em formato XLS.

Os seis datasets publicados em arquivos agregados ZIP exclusivamente, não têm a possibilidade de ser obtidos em algum outro formato que seja arquivo estruturado. Caso semelhante acontece com um dataset que está publicado unicamente como um grafo interativo. Esses datasets disponibilizados com formatos não estruturados correspondem com o $10 \%$ da amostra obtida no site da Colômbia.

A Figura 4 traz uma visão conjunta dos tipos de arquivos que usaram os dois países nos datasets encontrados. 


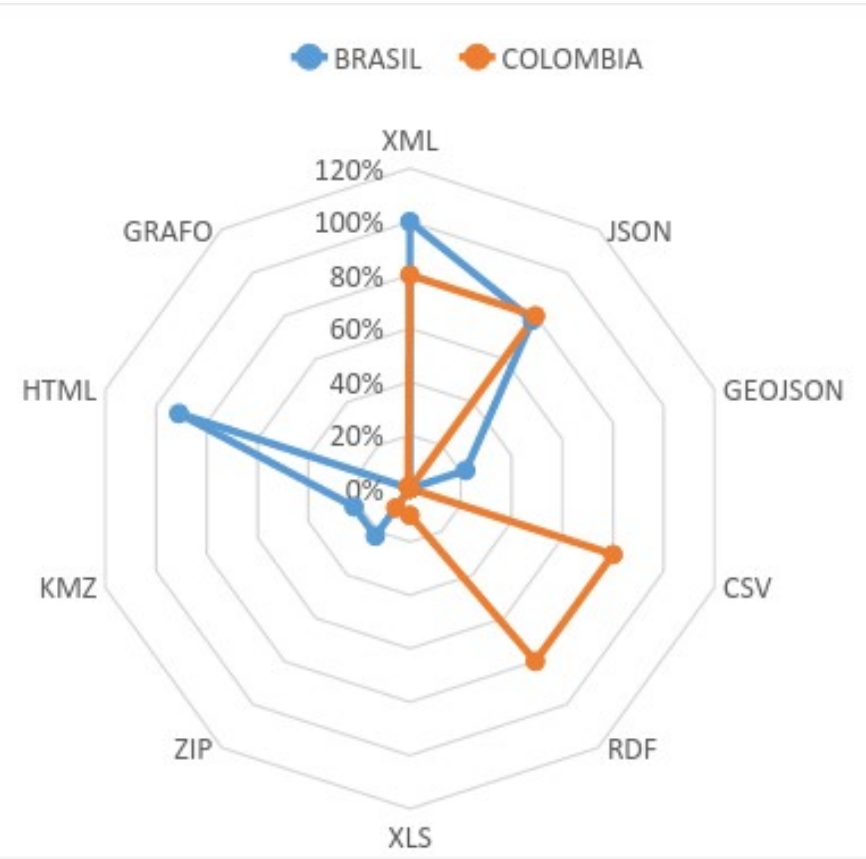

Figura 4. Visão conjunta dos tipos de arquivos usados nos dois países. Fonte: Elaborado pelos autores.

Considerando a classificação de arquivos presentes nos datasets, proposta no quadro II, verifica-se o uso predominante dos formatos XML e JSON nos dois países, embora em menor medida na Colômbia, apresentando um claro entendimento e assimilação no uso de arquivos estruturados que promovam processos automáticos nos dados disponibilizados. Apesar de que no Brasil não se observa o uso dos formatos CSV e RDF no domínio da pesquisa, o fato de ter o cem por cento em formato XML é um ponto de destaque desse país, que ainda apresenta o formato HTML como alternativa além do XML.

\section{Princípio Não Discriminatório}

Nenhum dos dois países têm restrições discriminatórias nos datasets analisados, isto é, não requerem registro dos usuários antes da consulta, nem durante dela, permitindo deste modo buscas anónimas.

\section{Princípio Não proprietário}

No caso do Brasil, a totalidade dos datasets analisados nesta pesquisa, estão disponibilizados em formato de arquivos não proprietários, de modo que nenhuma entidade ou instituição tem controle sobre o uso e acesso aos dados presentes nesses datasets. Entretanto na Colômbia, o 10\% dos datasets da amostra, se encontraram em formato Excel, desenvolvido pela multinacional Microsoft ${ }^{11}$. Esse formato de arquivo não proprietário é disposto de forma exclusiva, ou seja, não como alternativa para a escolha por parte dos usuários dos dados, senão como único formato desses datasets.

\section{Princípio Livre de Licença}

Nos datasets recuperados no site do Brasil, encontram-se dois valores no metadado Tipo de Licença: Licença Open Data Commons Open Database (ODbL) (Licença Aberta para Bases de Dados de Open Data); e, Licença Não Especificada.

Dos 32 datasets recuperados, o 78\% tem como tipo de licença a ODbL. Foi possível inferir que nos conjuntos onde não têm tipo de licença, os dados são de domínio público, entretanto, é preciso fazer específico tal tipo de licença.

No site de dados da Colômbia, observa-se que o 100\% dos datasets recuperados tem no metadado Permiso, o valor Público. Apresenta ainda, um metadado de nome Licencia, onde em dois datasets têm como valor relacionado o nome da entidade que está disponibilizando os dados.

Os dois sites de dados abertos não apresentaram uma definição da estrutura dos metadados a fim de que os usuários possam ter certeza do conteúdo que vai apresentar-se em cada atributo, para assim ter claridade nas implicações legais sobre o tipo de licença. 


\section{CONSIDERAÇÕES}

Salienta-se o fato de que tanto o Brasil quanto a Colômbia têm em andamento políticas públicas de Estado para a publicação de dados abertos de governo, evidenciadas nesta pesquisa com processos de disponibilização de datasets em sites administrados por órgãos de governos de cada país.

Observou-se que os dois países satisfazem as recomendações dos princípios de Sebastopol 4 e 6 , permitindo o acesso aos datasets sem discriminação relacionada a perfis de usuários, características tecnológicas desse acesso, registros prévios de usuários, nem filtros sobre quais os usuários ou as plataformas que podem acessar e consultar os datasets.

No caso da Colômbia tem tanto um número maior de datasets publicados quanto uma maior diversidade nos assuntos desses dados, porém, frente ao Brasil, ainda tem que apurar no fato de conseguir que os dados publicados estejam em formatos estruturados processáveis por máquina e em formatos de arquivos não proprietários, até alcançar o 100\% de datasets publicados com essas características, buscando assim satisfazer os princípios de Sebastopol 5 e 7.

$\mathrm{Na}$ amostra abordada na pesquisa percebeu-se que o Brasil tem assimilado corretamente as indicações técnicas sobre os formatos de representação de dados, quando apresenta a totalidade dos datasets da amostra em arquivos estruturados processáveis por máquina e em formatos não proprietários, contribuindo assim com os processos para o uso e reuso dos dados publicados. Nota-se ainda que no caso do Brasil, mesmo que o formato de arquivo HTML, formato que não atinge a necessidade de reutilização de dados, esteja utilizando-se em uma alta porcentagem dos datasets, ele é utilizado de maneira alternativa e não exclusiva, estando de modo concomitante a formatos tais como XML e JSON.

As visualizações gráficas dos dados têm que estar sempre acompanhadas dos datasets, os quais até podem estar visualizando-se como apresentação interativa, porém, sem substituir o objetivo de dispor os dados puros. Propõe-se que para casos como da Colômbia, não seja considerado o uso da opção de visualização gráfica dos datasets, como visualização exclusiva dos dados.

Visando o princípio 8, o metadado Tipo de Licença deve estar corretamente descrito, uma vez que está presente nos datasets, porém, sem estar normalizado. Isto faz com que o usuário tenha que pesquisar sobre as implicações legais em atividades como redistribuição dos dados, compilação, propagação, etc., além de interpretar particularmente os casos de licenças que não são especificadas, e em seu lugar, está indicando-se o nome do órgão de governo que está publicando esses dados.

Novas pesquisas poderão ser direcionadas para refletir sobre um modelo de avaliação dos primeiros três princípios de Sebastopol, assim como para aprofundar na avaliação dos princípios 4, 5, 6, 7 e 8 até em outros domínios do conhecimento. Sugere-se priorizar a reflexão, por parte de profissionais da Ciência da Informação e por grupos transdisciplinares de conhecedores da natureza dos dados que estão publicando-se, em torno da padronização e normalização dos metadados que caracterizam os datasets, bem como a análise de propostas para a avaliação da granularidade e o grau de processamento dos dados publicados.

Constatou-se que dados abertos sobre Agricultura e Desenvolvimento Rural estão sendo publicados nos países analisados. No Brasil, esses datasets disponibilizam dados relacionados com Agricultura Familiar, Seguros, Programa de Aquisição de Alimentos (PAA), Bibliotecas Rurais, Banco Nacional de Desenvolvimento Económico e Social (BNDES), Classificação Nacional de Atividades Económicas (CNAE) na Agricultura, CNAE Pesca, Programa de Fortalecimento da Agricultura Familiar (PRONAF), Infraestrutura Hídrica na Agricultura e dados sobre Irrigação. Na Colômbia, os datasets publicados contén dados sobre Acueductos, Agricultura, Cultivos, Usos de Suelos, Agropecuaria, Almacenes Agrícolas, Flora-Fauna, Asistencias Técnicas, Asociatividad, Avales Catastrales, Distribución de Veredas, Pecuaria, Formalización de tierras, Productores, Políticas Públicas, Inventarios de Fincas, Precios de Mercado Campesino, Restitución de Tierras, Propiedades Rurales, Unidad de Planificación Rural Agropecuaria (UPRA).

Considerando que no Brasil somadas as participações no Produto Interno Bruto (PIB) de atividades correlatas à agricultura e a mineração atingem o 6,8\%, sendo na Colômbia o 13\% do PIB, e que a porcentagem de exportações em cada país de produtos e serviços relacionados a esse setor, chegaram no ano 2016 a 26,9\% no Brasil e $15,8 \%$ na Colômbia (CEPAL, 2017), apontam-se possibilidades de trabalhos que contribuam, partindo da Ciência da 
Informação, no desenvolvimento de países, mediante estudos no escopo de ativos informacionais e as suas relações com avanços para regiões geopolíticas.

O anterior no estimado de que pesquisas acerca da disponibilização de dados abertos de governo, para a construção de ambientes democráticos, deverão ser caracterizadas por fluxos de informação eficientes (Rodrigues; Sant'Ana; Ferneda, 2015), e contribuir no fortalecimento da relação estado-cidadão (Garriga-Portolà, 2011).

\section{NOTAS}

${ }^{1}$ Disponível em: $<$ http://www.un.org/sustainabledevelopment/>.

2 Disponível em: $<\mathrm{http}: / /$ www.worldometers.info/>.

${ }^{3}$ Disponível em: <http://iipdigital.usembassy.gov/st/portuguese/article/2012/06/201206077052.html\#axzz4Ry7oygCG>.

4 A avaliação proposta pela OCDE analisa três áreas, a saber: disponibilidade dos dados, acessibilidade aos dados, e suporte governamental para o reuso dos dados (OCDE, 2017).

${ }^{5}$ A OGP buscou estabelecer compromissos concretos de parte de governos que estivessem interessados em estimular a disseminação aberta de suas ações com o fim de encorajar os cidadãos (OGP, 2017).

${ }^{6}$ Estados Unidos, Reino Unido, Noruega, Filipinas, Indonésia, África do Sul, Brasil e México (OGP, 2017).

${ }^{7} \mathrm{Na}$ atualidade 75 países são membros da OGP (OGP, 2017).

${ }^{8}$ Disponível em: $<$ https://upload.wikimedia.org/wikipedia/commons/4/4c/Diagn\%C3\%B3stico_de_abertura_de_dados.pdf $>$.

${ }^{9}$ Quatro condições da informação reutilizável: não existem barreiras técnicas para reutilizá-la; possibilidade de acesso automatizado; existência de esquema legal que permita o seu uso; e, acesso à estrutura da informação (Abella; Ortiz-de-Urbina-Criado y de-Pablos-Heredero, 2014).

10 Disponível em: $<$ https://pt.wikipedia.org/wiki/Proxy $>$.

${ }^{11}$ Disponível em: <https://products.office.com/pt-br/excel>.

\section{REFERÊNCIAS}

ABELLA, A. Full description of MELODA. Disponível em: < http://www.meloda.org/full-description-of-meloda $>$ [Consultado: 2 fev. 2018]

ABELLA, A.; ORTIZ-DE-URBINA-CRIADO, M. y DE-PABLOS-HEREDERO, C. MELODA métrica para evaluar la reutilización de datos abiertos. El profesional de la información, 2014, vol. 23, nº 6, p. 582-588.

ALVES, R.C.V.; SIMIONATO, A.C. y SANTOS, P.L.V.A.C. Aspectos de granularidade na representação da informação no universo bibliográfico. En Anais do I Encontro Nacional de Catalogadores e III Encontro de Estudos e Pesquisas em Catalogação. Florianópolis, 2012.

ATTARD, J. et al. A systematic review of open government data initiatives. Government Information Quarterly, 2015, vol. 32, no 4, p. 399-418.

BANCO MUNDIAL. Datos. Disponível em: <http://datos.bancomundial.org/indicator/AG.LND.AGRI.ZS> [Consultado: 5 abr. 2017]

BRASIL. Ministério do Planejamento, Orçamento e Gestão. Instrução Normativa no 4, de 12 de abril de 2012. Institui a Infraestrutura Nacional de Dados Abertos - INDA. Disponível em: <http://dados.gov.br/paginas/instrucaonormativa-da-inda $>$ [Consultado: 18 fev. 2017]

BRASIL. Presidência da República. Casa Civil. Lei $n^{\circ} 12.527$, de 18 novembro de 2011. Regula o acesso a informações previsto no inciso XXXIII do art. 5o, no inciso II do § 3o do art. 37 e no § 2o do art. 216 da Constituição Federal; altera a Lei no 8.112, de 11 de dezembro de 1990; revoga a Lei no 11.111, de 5 de maio de 2005, e dispositivos da Lei no 8.159, de 8 de janeiro de 1991; e dá outras providências. Disponível em: $<$ http://www.planalto.gov.br/ccivil_03/_ato2011-2014/2011/lei/112527.htm> [Consultado: 18 fev. 2017]

CEPAL. Bases de Datos y Publicaciones Estadísticas. 2017. Disponível em: $<$ http://estadisticas.cepal.org/cepalstat/Portada.html> [Consultado: 10 fev. 2018]

COLÔMBIA. Guía de datos abiertos en Colombia. Disponível em: $<$ http://estrategia.gobiernoenlinea.gov.co/623/articles-8248_Guia_Apertura_Datos.pdf $>$ [Consultado: 16 jun. 2016]

COLÔMBIA. Ley n ${ }^{\circ} 1712$, de 6 de marzo de 2014. Por medio de la cual se crea la ley de transparencia y del derecho de acceso a la información pública nacional y se dictan otras disposiciones. Disponível em: $<$ http://www.mintic.gov.co/portal/604/w3-article-7147.html > [Consultado: 16 jun. 2016]

COLÔMBIA. Open Data Readiness Assessment. Disponível em: $<$ http://estrategia.gobiernoenlinea.gov.co/623/articles-9407_evaluacion_apertura_datos.pdf $>$ [Consultado: 10 ago. 2016]

FAO e OCDE. Perspectivas Agrícolas 2015. Paris: OECD Publishing, 2015. 
GERTRUDIS-CASADO, M.C.; GÉRTRUDIX-BARRIO, M. y ÁLVAREZ-GARCÍA, S. Competencias informativas profesionales y datos abiertos: retos para el empoderamiento ciudadano y el cambio social. Comunicar: Revista Científica de Comunicación y Educación, 2016, vol. 24, nº 47, p. 39-47.

GONZALEZ-ZAPATA, F. y HEEKS, R. The multiple meanings of open government data: understanding different stakeholders and their perspectives. Government Information Quarterly, 2015, vol. 32, nº 4, p. 441-452.

HOWARD, A. No joke: open data fuels transparency, civic utility and economic activity [online]. Disponível em: $<$ http://gov20.govfresh.com/no-joke-open-data-fuelstransparency-civic-utility-and-economic-activity/> [Consultado: 1 fev. 2018]

HUIJBOOM, N. y VAN DEN BROEK, T. Open data: an international comparison of strategies. European Journal of ePractice, 2011, no 12, p. 1-13.

LAMPARELLI, R.A.C. Agricultura de precisão. Disponível em: <http://www.agencia.cnptia.embrapa.br/gestor/canade-acucar/arvore/CONTAG01_72_711200516719.html> [Consultado: 17 fev. 2017]

MÉXICO. Diagnóstico sobre el estado de preparación de datos abiertos: preparado para el Gobierno de los Estados Unidos Mexicanos. Disponível em: <http://opendatatoolkit.worldbank.org/docs/odra/odra_mexico_complete.pdf> [Consultado: 10 ago. 2016]

NARDO, L.A.S. Análise de cenários para auxílio à tomada de decisão no plantio mecanizado de cana-de-açúcar. Dissertação de mestrado, Universidade Estadual Paulista, 2015.

OCDE. Government at a Glance 2017. Paris: OECD Publishing, 2017. Disponível em: $<\mathrm{http}$ //dx.doi.org/10.1787/gov_glance-2017-en> [Consultado: 10 jan. 2018]

OGP. Open Government Partnership. 2017. Disponível em: <https://www.opengovpartnership.org/about/about-ogp> [Consultado: 1 fev. 2018]

ONU. Objetivos de desarrollo sostenible. Disponível em: <http://www.un.org/sustainabledevelopment/es/hunger/> [Consultado: 19 fev. 2017]

OPEN GOVERNMENT DATA. The annotated eight principles of open government data. Disponível em: $<$ https://opengovdata.org/> [Consultado: 1 jul. 2016]

OPEN KNOWLEDGE BRASIL. Diagnóstico de abertura de dados Open Data Readiness Assessment (ODRA) e Plano de ação para a implementação da política de dados abertos. Brasília: Governo do Distrito Federal, 2014.

OPEN KNOWLEDGE FOUNDATION. How to open data. Disponível em: < https://okfn.org/opendata/how-to-opendata/> [Consultado: 12 mar. 2017]

PERU. Open Data Readiness Assessment. Disponível em: <http://opendatatoolkit.worldbank.org/docs/odra/odra-perufinal.pdf $>$ [Consultado: 10 ago. 2016]

PESET, F.; FERRER-SAPENA, A. y SUBIRATS-COLL, I. Open data y linked open data: su impacto en el área de bibliotecas y documentación. El profesional de la información, 2011, vol. 20, n² 2, p. 165-173.

RANGEL-CH, J.O. y AGUILAR-P, M. Una aproximación sobre la diversidad climática en las regiones naturales de Colombia. En RANGEL-CH, J.O. (ed.). Colombia Diversidad Biótica I. Bogotá: Universidad Nacional de Colombia, 1995 , p. 25-77.

REPÚBLICA DOMINICANA. Open Data Readiness Assessment. Disponível em: $<$ http://opendatatoolkit.worldbank.org/docs/odra/odra_republica_dominicana.pdf $>$ [Consultado: 10 ago. 2016]

SANT'ANA, R.C.G. y RODRIGUES, F.A. Uso de modelos de dados multidimensionais para a ampliação da transparência ativa. LIINC em Revista, 2013, vol. 9, n 2, p. 469-487.

SANTARÉM SEGUNDO, J.E. Tecnologias de informação e comunicação para disponibilização de dados abertos em formato semântico. Ibersid, 2013, vol. 7, p. 33-40.

SANTARÉM SEGUNDO, J.E. Web semântica, dados ligados e dados abertos: uma visão dos desafios do Brasil frente às iniciativas internacionais. En Anais do XVI Encontro Nacional de Pesquisa em Pós-Graduação em Ciência da Informação. João Pessoa, 2015.

SANTOS, P.L.V.A.C. y SANTANA, R.C.G. Dado e granularidade na perspectiva da Informação e Tecnologia: uma interpretação pela Ciência da Informação. Ciência da Informação, 2015, vol. 42, nº 2, p. 199-209.

UBALDI, B. Open government data: towards empirical analysis of open government data initiatives. OECD Working Papers on Public Governance, 2013, $\mathrm{n}^{\mathrm{o}}$ 22. Disponível em: <http://dx.doi.org/10.1787/5k46bj4f03s7-en> [Consultado: 1 fev. 2018]

VERHULST, S.G. y YOUNG, A. Open data in developing economies toward building an evidence: toward building an evidence base on what works and how. New York: Governance Lab, New York University, 2017.

W3C. Manual dos dados abertos: governo. Disponível em: $<$ http://www.w3c.br/pub/Materiais/PublicacoesW3C/Manual_Dados_Abertos_WEB.pdf $>$ [Consultado: 10 mar. 2017]

W3C. Standards. Disponível em: <https://www.w3.org./standards> [Consultado: 10 mar. 2017]

YOUNG, A. y VERHULST, S. The global impact of Open Data: key findings from detailed case studies around the world. Sebastopol: O'Reilly, 2016. 